\title{
Defining and addressing the nutritional needs of populations
}

\author{
Ricardo Uauy* \\ Instituto de Nutricion y Tecnología de los Alimentos (INTA), University of Chile, Santiago, Chile, \\ and London School of Hygiene and Tropical Medicine, London, UK
}

\begin{abstract}
Objective: To examine the present methods used to define nutritional needs, and to analyse the intrinsic limitations of the reductionist chemical, biological and medical approaches to assess requirements. To establish the necessity to incorporate the complexities emerging from a broader understanding of the biological sciences as well as to include environmental and social dimensions in addressing nutritional needs.

Method: Examples of the limitations of current approaches and the implications of these in defining potential solutions and policy options to address present nutritional problems are presented and discussed.

Conclusion: The chemical and biological sciences have provided a strong base for nutrition and have been essential in establishing nutrition as a science with public health relevance. However, these approaches are clearly insufficient to address the main challenges that confront nutrition science now in the twenty-first century. There is a pressing need to include the social, economic and human rights aspects in order to define future policies that will secure the right to safe and nutritious food for all.
\end{abstract}

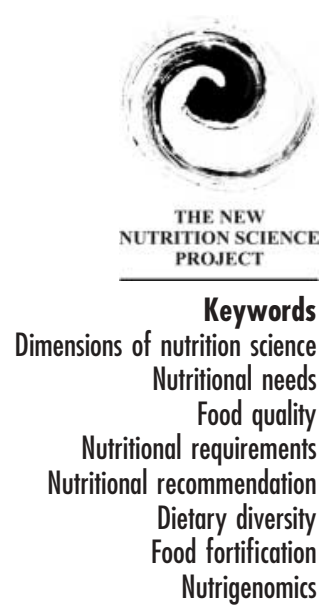

The nutritional needs of humans and the foods available to meet them have been intimately linked throughout evolution. Life on this planet evolved in an environment that provided energy and specific substrates to meet the trophic and reproductive needs of increasingly complex biological systems.

Thus biology from its origin was shaped by the available food supply while the presence of life influenced the place of various species in a given ecological niche. Heterotrophic life evolved based on the capacity of using autotrophic forms of life or other heterotrophic forms occupying a lower place in the food web as foods. On a biochemical basis we know that without plants that trap solar radiation and convert it into chemical energy, animal life as we know it would not be possible.

Humankind within the evolutionary tree/web has been unique in being able to gain from successive individual and social experiences in the selection of foods consumed and produced, processed and preserved. Humans as hunters, scavengers or gatherers were able to pass to others and to the next generation their dietary patterns including food preparations. Since the advent of agriculture, humankind has been in a position to define the foods produced in order to meet nutritional needs. More recently we are not only able to choose our foods from naturally occurring forms of life, but are now also able to design novel foods that have selective advantages in the production process or desired nutritional properties.
We are now at the threshold of a new era in which humans may in fact design their food supply to modify their life course and possibly their own evolution. The choices we make in the coming years may have profound influences in the future of humankind.

\section{Discussion}

\section{Human nutritional needs and foods available to meet them}

The foods produced since the advent of agriculture have evolved depending on prevailing environmental conditions that affect the climate, solar radiation, soil characteristics and water resources. These conditions, fundamental for the development of agriculture in prehistoric times, continue to play an important role in defining modern agriculture. Human diets have similarly changed from a predominantly gatherer-hunter or scavenger mode to the present agriculture-based model.

Humans in pre-agricultural times depended on foraging plant foods such as seeds, fruits and nuts as well as hunting small animals; if they inhabited the land/water interface they were able to collect molluscs and algae and catch fish. They were also likely to have been scavengers of meat and fat protected by bone (such as brain and marrow), left over from the hunt by predators larger and stronger than humans. Agriculture evolved in very specific ecological settings that facilitated the domestication and selection of 
The chemical and biological sciences have provided a strong base for nutrition and have been essential in establishing nutrition as a science with public health relevance. However, these approaches are clearly insufficient to address the main challenges that confront nutrition science now in the twenty-first century. There is a pressing need to include the social, economic and human rights aspects within an ethical framework, in order to define future policies that will secure the right to safe and nutritious food for all.

the four main crops upon which we relied for our food supply. In these settings, wheat, rice, corn and potatoes became the key foods to support the expansion of human populations to the current level of over 6 billion individuals ${ }^{1,2}$

Traditional dietary patterns have changed with time and have withstood the test of human evolution. Indeed, most naturally occurring dietary patterns meet or exceed the nutritional needs of populations, although this is not the case where social or economic conditions limit access to food (purchasing capacity) or where cultural practices restrict the choice of foods consumed. However, within the framework of our present understanding of foodhealth relationships, it seems likely that a large variety of foods can be combined in varying amounts to provide healthy diets. Thus, it is difficult to determine a precise indispensable intake of individual foods that can, when combined with other foods, provide nutritionally adequate diets under all conditions. Perhaps the exception that proves this rule is human milk, now accepted as a source of complete nutrition for the first 6 months of life, provided enough sun exposure is allowed to prevent vitamin D deficiency.

The prevailing view is that a large set of food combinations is compatible with nutritional adequacy, but that no given set of foods can be extrapolated as absolutely required or sufficient across different ecological settings. Recent trends in the globalisation of food supplies provide clear evidence that dietary patterns and even traditionally local foods can move across geographical niches ${ }^{2}$.

The modern approach in defining nutritional adequacy of diets and dietary recommendations has progressed over the past two centuries in accordance with the scientific understanding of the biochemical and physiological basis of human nutritional requirements in health and disease. The definition of essential nutrients and nutrient requirements has provided the scientific underpinnings for nutrient-based dietary recommendations. However, there are obvious limitations to the reductionist nutrient-based approach, since people consume foods and not nutrients. Moreover, the effect of specific foods and dietary patterns on health goes well beyond the combination of essential nutrients the food may contain. For example, if we neglect to integrate bioavailability or nutrient interactions in defining trace element recommendations, we will not be able to assess the true nutritional value of foods ${ }^{2,3}$.

In addition, factors unrelated to diet commonly play a key role on the health effect of diets; for example, parasitic infections rather than iron deficiency may be the cause of anaemia in many parts of the world. Similarly, if we continue to ignore or undervalue the key role of physical activity in achieving energy balance, dietary recommendations will fail to meet the goal of preventing obesity and other nutrition-related chronic diseases.

\section{Significance of changing approaches used in defining nutritional needs}

Criteria used to estimate nutritional needs have changed over time. Different approaches have had major implications in defining the magnitude of nutritional problems as well as the proposed solutions to them ${ }^{2,3}$. Four main approaches have been used in the past, these are:

\section{Clinical approach}

This is the intake to cure or prevent manifestations of disease. The values derived using the need to cure or prevent disease are usually much higher than those required to maintain health. In the case of protein, needs to cure deficit are three times greater than those to preserve health, even in young children. The value of this approach is its intrinsic validity, no one would argue on the nature of the outcome.

\section{Customary consumption}

This is the intake observed in populations that are 'apparently healthy'. This approach is not very precise, it provides a wide range of acceptable values since humans can be in good health across a wide range of intakes, and for some nutrients the upper value of the range can be up to 10 times the lowest observed value. It is still used when no other information on sufficiency is available.

\section{Functional adequacy}

This approach is based on assessing function; that is, defining nutritional sufficiency using molecular, biochemical, physiological or immunological indices related to the intake of specific nutrients. This is the most commonly used method since it permits the definition of a dose response. The key problem when using 
functional indices is the sensitivity and specificity of the indicators; additionally, the use of indicators requires that we have a well-defined outcome related to the specific nutrient we would like to assess.

\section{Optimal nutrient intake}

This is the intake associated with optimal physical and mental health. The question 'Optimal for what?' is usually answered by the suggestion that diets or specific nutrients can improve physical and mental performance, enhance immunity, prevent cancer, or add healthy years to our life. This concept is commonly abused and usually unsupported by appropriate population-based cohort or randomised controlled studies. A detailed analysis of the relative merits of these criteria is beyond the scope of this paper. The present challenge is defining optimal in terms of long-term health in a manner that can be practically assessed at various times in the life course.

The quantitative definition of nutrient needs and their expression as recommended nutrient intakes (RNIs) or dietary allowances (RDAs) have been important instruments of food and nutrition policy in many countries and have focused the attention of international organisations. These are customarily defined as the intakes of energy and specific nutrients necessary to satisfy the requirements of a group of healthy individuals. Over the past five decades, scientific expert committees have established nutrientbased recommendations for virtually every essential nutrient.

This nutrient-based approach has served well to advance science, but has not always fostered the establishment of nutritional and dietary priorities consistent with broad public health interests at national and international levels ${ }^{2-4}$.

In fact, as judged post facto, nutrient-based recommendations may have misguided some efforts to solve key nutritional problems. For instance, the emphasis on protein - both quantity and quality, derived from studies of single food sources evaluating the effect on growth rates of young animals - placed a premium on the development of animal foods (meat, eggs and cow's milk) and failed to include the concept of amino acid complementarities of cereal-legume mixes ${ }^{5-7}$. McLaren was the first to draw attention to this misguided development ${ }^{8}$.

In fact, when human infant and adult studies were conducted several decades later the nutritional value of mixed diets from around the world was similar to that of animal proteins except for a small increase in nitrogen lost in stools when mixed vegetable protein sources were consumed. Thus the protein gap, that led key scientists at the time to demand urgent international action to address it, was averted not by global initiatives but by a change in the experimental models to assess protein quality for humans $^{5-7,9}$ (see Box 1).

\section{Meeting nutritional needs of populations given diversity in individual needs}

The possibility of defining nutrient requirements and actually dietary recommendations based on individual needs is indeed attractive, considering that optimal diets may in fact be specific for a given genotype. The contrasting argument accepts that the optimal diet may in fact be different for each individual; yet at the same time it recognises that genetic differences need not necessarily imply different dietary guidelines ${ }^{2}$.

The only justification for genotypically defined diets would be if there was a solid basis for genetic individuality, with significant health consequences for each genotype. Present knowledge on genomics indicates that close to 30000 genes encode the biological basis that defines Homo sapiens as a distinct species, but apparently only about 3000 of these define key health or disease conditions. Mutations in these 3000 genes occur infrequently (1 to 0.01 per 1000 births); some of these will modify nutrient requirements and may define nutritional individuality requiring special diets. Nobody would argue that people who are not able to oxidize phenylalanine, PKU (phenylketonuria) patients, require low or phenylalanine-free diets and need to be protected from excessive intake of this amino acid (consider the label in aspartame sweeteners warning persons with PKU on the danger of this exposure).

Others cannot absorb zinc efficiently and thus require an intake several times the normal recommendation to maintain health. In still others copper may become toxic at common levels of intake, as is the case of Wilson's disease patients. However, since these mutations are rare and occur similarly across different regions of the world, their existence does not support the need to establish different nutritional recommendations across genotypes but rather to identify and protect the very few genetically susceptible individuals with special needs.

More recently we have begun to unravel the significance of changes in a given base pair of the DNA strand, so-called single nucleotide polymorphisms (SNPs). These occur in approximately 1 per 1000 base pairs, and while in most cases SNPs are silent, in other cases they can affect the expression of one or more genes and thus may have major consequences in nutrient metabolism. The concept of biochemical individuality coined by Garrod a century ago ${ }^{10}$ acquires new meaning with the understanding of the intricate nature of gene expression and the interaction between genes and SNPs.

Presently most agree that there exist close to 15 million distinct SNPs, and it is these that make us truly unique. It remains to be seen if biochemical/genomic individuality leads to nutritional individuality; if this proves to be the case we may need to redefine the approach used to establish dietary recommendations. Examples of genetic polymorphisms that confer specific nutritional needs based on current knowledge are presently around 100; 
this list will surely grow. For some, as is the case for the enzyme MTHFR (methyltetrahydrofolate reductase), we already have evidence from meta-analysis of supplementation trials that these genotypically defined individuals require several times more folate than usually recommended to minimise risk of cardiovascular disease. In this case, this level of specificity has public health relevance ${ }^{2,11}$.

Populations in impoverished countries, where nutritional deficiencies still predominate, are ill prepared to respond to the complexity of genetic diversity. We need to first adequately face the problem of how to provide sufficient nutrients for those who are getting too little, without inducing excess in those who are getting enough, before addressing genetic individuality.

Eventually we will need to provide diets that will maximise health benefits and prevent nutrition-related chronic disease across individuals. This may be possible for some but not for all nutrients. At this stage we are just beginning to discover the implications of genetic and epigenetic influences on nutritional needs of individuals and population groups. Unless the genetic factor(s) define(s) a special nutritional need that is linked to a strong susceptibility for a given health disorder, we should

\section{Box 1 - Energy needs and optimal growth of infants and young children: a changing paradigm}

The traditional definition of optimal growth has been 'more is better'; this implies that heavier and faster is preferred over lighter and slower. This in fact might be true if the relevant public health issues were underweight and malnutrition. However, in the present scenario where overweight and obesity prevail, we need to consider long-term health as the relevant outcome to assess early growth. This paradigm shift has clear implications in the assessment of energy needs of children. Energy intakes observed in industrialised countries, where children grew fast and heavy, served to define recommended energy intakes in the recent past.

The present approach, based on defining needs from energy expenditure and energy stores to maintain health ${ }^{1}$, better serves the need to address the global epidemic of obesity. For populations in most middleand low-income countries, providing energy intakes based on consumption patterns of children living in high-income countries will only contribute to advancing the obesity problem.

The latest Food and Agriculture Organization/World Health Organization (WHO)/United Nations University (UNU) 2004 report $^{1}$ notes that the energy needs of children have been systematically overestimated by $10-25 \%$. Present data from infants under 1 year of age, using energy expenditure estimates from doubly labelled water, reveal that for this group the overestimation has been close to 20\%. Furthermore, the current estimates of energy needs for breastfed infants are around $7 \%$ below those of formula-fed infants.

These differences may appear to be of small magnitude but if the old recommendations are systematically used in the feeding of children today, they can effectively serve to promote obesity in the early years of life and affect long-term health ${ }^{1-4}$. We are just now realising that normality needs to be defined by favourable nutrition and health outcomes across the life span and not based solely on observations of apparently healthy populations. Thus, normative data on recommended protein and energy intakes for the first 6 months of life have been redefined based on the intakes of exclusively breastfed infants. The growth and metabolic indices of the breastfed infant are now considered the 'gold standard' or reference to define the nutritional needs of young infants. Similarly, the new UNU/WHO ${ }^{5}$ growth reference, due to appear in 2005 , is based on a prescriptive feeding model of predominantly breastfed infants for the first 4-6 months of life.

We, as scientists, have the obligation to critically examine not only the evidence but also the appropriateness of the design of studies used to obtain it. As with many things in biology, the context in which the experiment is carried out defines the answer that is obtained. The colour of things depends on the colour of the crystal you use to gauge them.

\section{References}

1 Food and Agriculture Organization of the United Nations (FAO). Human Energy Requirements. Report of a Joint FAO/World Health Organization/United Nations University Expert Consultation. FAO Technical Report Series No. 1. Rome: FAO, 2004.

2 Food and Agriculture Organization of the United Nations (FAO)/World Health Organization (WHO). Energy and Protein Requirements. Report of a Joint FAO/WHO Ad Hoc Expert Committee. FAO Nutrition Meetings Report Series No. 52; WHO Technical Report Series No. 522. Rome/Geneva: FAO/WHO, 1973.

3 World Health Organization (WHO). Energy and Protein Requirements. Report of a Joint Food and Agriculture Organization of the United Nations/WHO/United Nations University Expert Consultation. Technical Report Series No. 724. Geneva: WHO, 1985.

4 Bhargava S, Singh H, Fall C, Osmond C, Lakshmy R, Barker D, et al. Relation of serial changes in childhood body mass index to impaired glucose tolerance in young adulthood. New England Journal of Medicine 2004; 350: $865-75$.

5 Garza C, de Onis M. Rationale for developing a new international growth reference. Food and Nutrition Bulletin 2004; 25: 5-13. 
not use genomics to shape nutritional recommendations ${ }^{11}$. However, this position will most likely change as we increase our ability to detect these genetic conditions and be in a position to do something about them.

Present-day humans may in fact have the unique responsibility of defining the food/nutrient supply that may contribute in shaping human evolution in years to come. The spectre of a man-made turning point in human evolution may seem far-fetched, yet nutrition in the past without doubt has been a significant factor in defining the evolution of all species including primates. We should face this responsibility considering not only the Hippocratic dictum primum non nocere, but also reaffirming our commitment to the well-being of humankind as a whole.

\section{Establishing nutritional recommendations: prevent deficit while avoiding excess}

The criteria used to define individual requirements include a gradient of biological effects related to the level of nutrient intake. This dose response is assumed to have a Gaussian distribution (bell-shaped); thus a sigmoidshaped function of deficit and excess can be derived at low and high intakes respectively, rendering a U-shaped risk function. Thus a valley of variable width representing the range for optimal intake is defined. The evaluation of adequacy of nutrient intakes of populations requires good quantitative information on the distribution of usual nutrient intakes within the population as well as knowledge of the distribution of requirements ${ }^{2,3}$.

It is clearly inappropriate, despite being frequently done, to examine mean values of population intakes and take the proportion that falls below the daily recommended intake to define the population at risk of inadequacy. A more complex issue arises when the population needs are not normally distributed, or there are two or three sub-populations within the apparent single bell-shaped curve. In many situations we may be faced with having to increase the intake of a population subgroup while having to decrease the intake of a different subgroup. We must be ready to reconsider the single Gaussian distribution of nutrient needs ${ }^{2,3}$.

In fact, as we fortify staple foods we may find that there may be population subgroups susceptible to nutrient excess. For example, it has been estimated that perhaps $10 \%$ of the population is heterozygous for haemochromatosis, a genetic condition where iron absorption is enhanced, and in the homozygous condition iron accumulation throughout life leads to permanent liver damage. The possibility that those with a single abnormal gene may also accumulate iron with adverse consequences in terms of degenerative disease has been raised.

We may find that iron supplements administered to populations with a high prevalence of anaemia but with a concurrent heavy infectious load may in fact aggravate infection and be deleterious, since some microbes also benefit from the extra iron. A recent population-based iron supplementation intervention in an economically developing country had to be stopped because infants in the iron-supplemented group had increased mortality due to infection. We will need to revisit Paracelsus, who almost 500 years ago indicated that poison or remedy is defined by the dose that we take rather than by the nature of the substance.

\section{The challenge of access to food of adequate quality}

A healthy diet can be attained in more than one way, given the variety of foods that can be combined. In practice, the set of food combinations that are compatible with nutritional adequacy is restricted by the level of food production that is sustainable in a given ecological and population setting. In most countries this restriction has been overcome, since imported food can provide for a stable food supply independent of local food production. Of greater significance are the economic constraints that limit food supply at the household level; these are frequently the true underlying causes of nutritional deficiencies.

Populations in impoverished countries often consume a monotonous diet out of need rather than choice, as their access to different foods is curtailed by economic factors. The percentage increase in the consumption of a food item when income increases by $1 \%$ is called the elasticity of consumption of the given food item. Most staple foods such as rice, wheat and corn have low income elasticity, meaning that even if income increases greatly, the increase in the amount of staple foods eaten will be small ${ }^{2}$. However, meat and other animal food products have high income elasticity, meaning that there is a large effect of income on consumption patterns. This is illustrated by the fact that the amount of animal protein foods consumed by the wealthiest $20 \%$ of the world's population is four times greater than that consumed by the poorest $20 \%$; while for cereal intakes, the differences are negligible ${ }^{2,3,11}$.

If access to food was not dependent on income but on need, the food available globally would be sufficient to meet the demand of humankind. A corollary to this conditional statement is that unless economic constraints in food consumption are overcome, dietary diversification will fail. The prevention of malnutrition of women and children through dietary means in economically deprived population groups will not work unless people have access to foods that are adequate in quantity and quality. This comprises the human rights dimension of meeting human nutrition needs: the right to food. Food security cannot be determined based on the availability of food energy alone: nutritional security requires that all essential micronutrients be covered by the food supply.

\section{How to accomplish dietary diversity in practice}

It is essential to work on strategies that promote and facilitate dietary diversification among the poor to achieve the complementation of cereal/tuber-based diets with 
micronutrient-rich foods such as legumes, vegetables and fruits, and some animal foods. If we examine the presence of food subsidies in rich countries and the tariffs imposed on agricultural products from poor countries, we can understand why the structure of world trade favours a net flow of nutrient-dense products from poor to rich countries and why a cow in the European Union has greater access to food than the 2 billion inhabitants of the planet living on less than \$US 2 a day. In the absence of major changes in income distribution in poor countries, or major accelerations in economic growth in most of the economically developing world, other possible alternatives to achieve adequate dietary intake must be sought.

Presently, fortification of staple foods is the most commonly used strategy to enhance specific nutrient density of foods, especially when food-based approaches are not feasible due to economic constraints. Fortification refers to the addition of nutrients beyond its natural content to a commonly eaten food. It can be single, with only one nutrient (fortificant) added, or multiple, to include two or more nutrients.

Fortification should be seen as complementary to food-based strategies and not as a replacement to dietary diversification, and can serve as a cost-effective measure to resolve micronutrient deficiencies until food-based approaches become feasible. Food-based approaches which require access to sufficient highquality, nutrient-dense foods can often take longer to achieve, but once established, are generally sustainable $e^{2,3}$.

\section{Modification of plant and animal foods to meet buman nutritional needs}

The advent of new agricultural practices including improvement of soils and enhanced plant micronutrient

\section{Box 2 - A vision and mission for the International Union of Nutritional Sciences}

The challenge for the International Union of Nutritional Sciences (IUNS) for this century and immediately, for the remaining years of this first decade, is to integrate biological, social and environmental dimensions into its work in order to become a truly global union, meaning that more leadership will need to come from Africa, Asia and Latin America; and to train young nutrition scientists to become our future leaders.

This implies an overall ethical framework, awareness of evolution and history, and application of broad principles including those of human rights and the sustained protection of human, living and physical resources. In turn, this requires that our profession becomes more aware of and sensitive to global social and environmental changes and their impact on the nutrition and health of humankind.

\section{Vision}

To live a life without malnutrition is a fundamental human right. The persistence of malnutrition, especially among children and mothers, in this world of plenty is immoral. Nutrition improvement anywhere in the world is not a charity but a societal, household and individual right ${ }^{1}$.

It is the responsibility of the world community to find effective ways and means to invest in better livelihoods and to avoid future unnecessary social and economic burdens. With collective efforts at international, national and community levels, ending malnutrition is both a credible and an achievable goal $^{1}$.

\section{Mission}

To promote advancement in nutrition science, research and development through international co-operation at the global level.

To continue in the path of progress we need a stronger union. The IUNS is the one and only truly global nutrition community. Its mission includes:

- To provide scientific and moral leadership to address global nutrition and food problems, mobilising the nutrition scientific community locally, nationally and at the regional level, in order to create the conditions necessary for sustained action to secure the right to safe and nutritious food for all.

- To integrate the many views under-represented in the global debate on the science and practice of nutrition. Leaders from China, India and other parts of Asia need to join Africans and Latin Americans, and those from the industrialised world, in raising awareness and demanding and leading actions to eliminate malnutrition in all its forms as a constraint on human and social development.

- To support the training of the next generation of nutrition scientists as leaders in both the science of nutrition and its practice in food and nutrition policies and programmes, as advocates, communicators and as agents of change, in order to sustain the achievement of this mission and vision.

\section{Reference}

1 James WPT, Smitasiri S, Ul Haq M, Tagwiyeri J, Norum K, Uauy R, et al. Ending malnutrition by 2020 - an agenda for change in the millennium. WHO Food and Nutrition Bulletin 2000; 21S: 1S-76S. 
content via classical plant breeding or genetic modification may also enhance the potential impact of food diversification. These processes may provide new meaning to effective fortification for key micronutrients, since it may be possible to include bioavailable micronutrients into crops that are presently being consumed as staple foods.

Recent developments in genetic modification offer great promise in achieving multiple micronutrient sufficiency from staple foods. For example, the introduction of rice varieties rich in iron-containing proteins and carotene or retinol may drastically change the way we approach prevention of iron and vitamin $\mathrm{A}$ deficits. The elimination of anti-nutritional factors that affect bioavailability of minerals, either through traditional breeding or genetic modifications, may also enhance the utilisation of iron and zinc in regular plant foods.

The nutritional quality of animal foods can also be affected by production practices. For example, the type and quantity of fats present in monogastric animals may be determined by the feed provided; thus if pigs are given feed sources rich in oleic acid or $n-3$ fatty acid their meat and fat acquire beneficial properties in comparison to those given the standard diet. Similarly, poultry that are raised free-ranging will have lower total fat and more $n-3$ fatty acids than if they are given a maize-based feed and constrained in their movements. Eggs can be enriched in long-chain $n-3$ fatty acids if animals are provided with fish meal or flax seed in their feed. Milk and meat from ruminants are more difficult to alter through diet since microbial fermentation in the rumen destroys most of the unsaturated fat fed.

However, new techniques of micro-encapsulation permit the delivery of nutrients beyond the rumen. Cattle living in constrained environments and given cereal-based diets have more fat in their flesh than their grass-fed counterparts. Genetic modification also allows drastic changes in fatty acid composition of animal tissues. Recently, the introduction of an $n-3$ desaturase gene from a round worm (the nematode Caenorbabditis elegans) into mice produced a dramatic increase in the DHA (docosahexaenoic acid) content of milk and muscle from these mammals ${ }^{12}$. DHA and other long-chain $n-3$ fatty acids are present in fish and human milk; they have important beneficial properties for vascular health and brain function across the life course.

Novel methods such as these provide unique ways to enhance the nutritional quality of diets without having to drastically modify consumption patterns. These approaches may prove acceptable to food producers who are reluctant to change their traditional food production systems, and will allow consumers to maintain their customary diet while achieving desirable changes in nutrient intake.

The key to the acceptance of novel foods by consumers will be a fully transparent process for safety and efficacy evaluation. In addition, the asymmetry concerning who benefits and who faces the potential risks, if any, needs to be addressed. Past experience in this area has been confounded by clear evidence of gains to producers with no benefit or even potential risks, due to insufficient evaluation, for consumers.

\section{Should we have global nutritional recommendations and dietary guidelines?}

The possibility of defining one set of dietary guidelines is indeed attractive, considering the need for uniformity in the global village and the potential economic benefits to producers and perhaps to consumers of having a common regulatory framework. Why should the optimal diet be different from one population to the next? Do we need different nutrition labels on sodium and fat quality in different countries? Cultural and/or ethnic differences may result in the selection of population-specific foods to meet human nutritional needs. Yet these do not necessarily imply different dietary guidelines and even less, different regulations

The only justification for national- or ethnic-based dietary guidelines would be if there was a solid genetic basis for nutritional individuality. As indicated above, present knowledge of the biological basis to support genetic- or even ethnic-specific nutritional recommendations is limited, especially if we restrict genetic differences to those of public health relevance. Universal guidelines may be desirable but they present new problems and novel challenges that we must face. A single unified set of guidelines will fail to address cultural diversity and the complex social, economic and political interactions between humans and their food supply ${ }^{2,3}$.

Take the example of calcium in the context of a global recommendation for calcium intake. Current recommendations are based mostly if not exclusively on data in Europe and North America. Moreover, study populations are predominantly postmenopausal women of Caucasian origin. Thus, data are clearly not truly representative of populations of different origins. This is of significance in light of the demonstrated difference between ethnic groups in markers of bone health such as the prevalence of osteoporosis: white Caucasian subjects have considerably higher rates of osteoporosis or fracture than black subjects, even within the same country. It has therefore been suggested that there may be genetic differences between population groups that modulate bone density and thus fracture rates. There is a known causal relationship between calcium absorption and vitamin D status (originating from diet or from the effect of ultraviolet light on the skin). Variation in the vitamin D status of population groups is dependent on exposure to sunlight; this varies according to latitude, i.e. with distance from the equator.

Current international recommendations for dietary vitamin $\mathrm{D}$ are again based almost exclusively on data 
collected in high-income country settings ${ }^{2}$. Moreover, the epidemiological data indicate a positive correlation between dietary calcium intake and the prevalence of fractures - the so-called "calcium paradox'11. There is also a sizeable variation in fracture rates within population groups supporting the suggestion that while genetics may prove important, physical activity and dietary factors also play crucial roles in determining bone health. Within populations, factors such as wealth, protein and isoflavone intakes and degree of solar radiation significantly affect markers of calcium status.

The present-day intakes of calcium in Africa and Asia would need to be doubled in order to meet the standards defined for North America. This would have major implications for our food supply; milk production would need to be increased since dairy products are one of the best sources of bioavailable calcium. Based on analysis of the complex interaction between dietary factors, calcium status, vitamin D status and the emerging information on the role of vitamin $\mathrm{D}$ receptor gene polymorphism in determining bone health, it is becoming progressively evident that it may not be desirable or scientifically valid to give global recommendations for calcium and vitamin D intakes $^{2}$.

Are unified guidelines achievable? The answer is that, for some nutrients, universal guidelines are certainly possible. However, as illustrated in the example of calcium and vitamin $\mathrm{D}$, we cannot have a universal recommendation based on data derived predominantly from postmenopausal women from Europe and North America. Dietary guidelines can most likely be harmonised following a unified approach to define them, but there must be room to accommodate environmental variables that define nutritional and metabolic characteristics as well as the specific epidemiological situation of a given society. There is also a need to incorporate cultural and social determinants of food choices that are ethnic-specific.

Global guidelines will fail unless they provide the necessary options for individuals and societies to select the foods they prefer, and combine them in the way that best suits their taste and preferences. Most consumers will agree that food is far too important to be left solely in the hands of nutrition experts.

\section{Conclusion}

The chemical and biological bases of nutrition science have contributed greatly to our understanding of the problems we presently face in addressing human nutritional and food needs. However, the global epidemiological profile facing us requires a systems approach well beyond the single or even multiple nutrient interventions characteristic of the programme and policy discussions of today.
The biochemical and clinical frameworks constrain the possibilities of a broader community/socially based approach in achieving food and nutritional sufficiency. The most important and urgent issues that confront food and nutrition scientists in the twenty-first century are beyond the scope of conventionally defined human biology. We must be willing to encompass the social, economic, political and human rights dimensions of nutrition, both to resolve the pending nutritional deficiencies as well as to address the global epidemic of nutrition-related chronic disease affecting humanity. A broader approach will permit us to advance in addressing the true challenge ahead, which is nutrition for optimal health and well-being at all stages of the life course.

\section{References}

1 Leitzmann C. Wholesome nutrition: suitable diets for the new nutrition science. Public Health Nutrition 2005; 8(6A): 753-9.

2 Uauy R, Hertrampf E, Dangour AD. Food-based dietary guidelines for healthier populations: international considerations [Chapter 107]. In: Shils ME, Ross CA, Shike M, Caballero B, Weinsier RL, Cousins JC, eds. Modern Nutrition in Health and Disease, 10th ed. Baltimore, MD: Williams \& Wilkins, 2005; in press.

3 Food and Agriculture Organization of the United Nations (FAO)/World Health Organization (WHO). Preparation and Use of Food-based Dietary Guides. Report of a Joint FAO/WHO Expert Consultation, Nicosia, Cyprus. WHO/NUT/96.6. Geneva: WHO, 1996.

4 Nestle M. Food Politics: How the Food Industry Influences Nutrition and Health. Berkeley, CA: University of California Press, 2002.

5 Food and Agriculture Organization of the United Nations (FAO)/World Health Organization (WHO). Energy and Protein Requirements. Report of a Joint FAO/WHO Ad Hoc Expert Committee. FAO Nutrition Meetings Report Series No. 52; WHO Technical Report Series No. 522. Rome/Geneva: FAO/WHO, 1973.

6 World Health Organization (WHO). Energy and Protein Requirements. Report of a Joint Food and Agriculture Organization of the United Nations/WHO/United Nations University Expert Consultation. Technical Report Series No. 724. Geneva: WHO, 1985.

7 Rand WM, Uauy R, Scrimshaw NS, eds. Protein-Energy Requirements in Developing Countries Results of Internationally Coordinated Research. Tokyo: United Nations University Press, 1984.

8 McLaren DS. The great protein fiasco. Lancet 1974; 2: 93-6.

9 James WPT, Smitasiri S, Ul Haq M, Tagwiyeri J, Norum K, Uauy R, et al. Ending malnutrition by 2020 - an agenda for change in the millennium. WHO Food and Nutrition Bulletin 2000; 21S: 1 S-76S

10 Garrod A. The incidence of alkaptonuria: a study in chemical individuality. Lancet 1902; 2: 1616-20.

11 World Health Organization (WHO). Diet, Nutrition and the Prevention of Chronic Diseases. Report of a Joint WHO/Food and Agriculture Organization Expert Consultation. Technical Report Series No. 916. Geneva: WHO, 2003. Also available at www.who.int.hpr

12 Kang JX, Wang J, Wu L, Kang ZB. Transgenic mice: fat-1 mice convert $n-6$ to $n-3$ fatty acids. Nature 2004; 427: 504 . 